\title{
Jejunal Dieulafoy Lesion Detected by Intraoperative Endoscopy in a Child
}

\author{
Hsun-Chin Chao, ${ }^{1,}$ Shao-Hsuan Hsia, ${ }^{2}$ Yung-Ching Ming, ${ }^{3}$ and Chuen Hsueh ${ }^{4}$ \\ ${ }^{1}$ Division of Gastroenterology, Department of Pediatrics, Chang Gung Memorial Hospital, Chang Gung University College of Medicine, Taoyuan City, Taiwan \\ ${ }^{2}$ Division of Pediatric Critical Care and Emergency Medicine, Department of Pediatrics, Chang Gung Memorial Hospital, Chang Gung University College of Medicine, Taoyuan \\ City, Taiwan \\ ${ }^{3}$ Department of Pediatric Surgery, Chang Gung Memorial Hospital, Chang Gung University College of Medicine, Taoyuan City, Taiwan \\ ${ }^{4}$ Department of Pathology, Chang Gung Memorial Hospital, Chang Gung University College of Medicine, Taoyuan City, Taiwan \\ "Corresponding author: Hsun-Chin Chao, Associate Professor, Division of Pediatric Gastroenterology, Department of Pediatrics, Chang Gung Children's Medical Center, Chang \\ Gung Memorial Hospital, Chang Gung University College of Medicine, 5 Fu-Hsing St., Guishan District, 33305, Taoyuan City, Taiwan. Fax: + 886-33288957, E-mail: \\ chaohero@yahoo.com
}

Received 2016 July 01; Revised 2016 August 03; Accepted 2016 October 13.

\begin{abstract}
Introduction: Dieulafoy lesion is an unusual cause of gastrointestinal bleeding in which significant, and often recurrent, hemorrhage occurs from a pinpoint non-ulcerated arterial lesion. It is usually located in gastric fundus, and is extremely rare in children.

Case Presentation: In this study, we present a male adolescent with jejunal Dieulafoy lesion. The patient was admitted to the hospital due to hematochezia and hypotension. No active bleeders were identified on endoscopy, colonoscopy, and double-balloon enteroscopy. Computed tomography angiography revealed active bleeders in jejunum. An intraoperative enteroscopy, using flexible endoscopy, revealed a jejunal Dieulafoy lesion with spurting bleeding. The patient was successfully treated by bowel resection.

Conclusions: Intraoperative endoscopy is a relatively efficient and safe modality for evaluating the bleeding Dieulafoy lesion in the small intestine, and is essential for directing surgery.
\end{abstract}

Keywords: Dieulafoy Lesion, GI Bleeding, Jejunum, Intraoperative Endoscopy, Child

\section{Introduction}

Dieulafoy lesion (DL) is mucosal erosion into a caliberpersistent submucosal artery (1). The lesion is named after G. Dieulafoy, who initially reported it in 1898 . DL is a rare cause of gastrointestinal (GI) hemorrhage, reported to be around 1-2\% of acute or chronic episodes of GI bleeding (2). It usually causes massive GI bleeding and is difficult to diagnose (2,3).

DL is typically located in the gastric fundus, and it usually occurs in adults, especially in the males and the elderly (3-5). It is an extremely rare source of GI bleeding in children. According to the reports, DL may occur as a possible congenital cause in children (6).

Here, we report an actively bleeding jejunal DL in a male adolescent. The case was finally diagnosed by intraoperative endoscopy and successfully treated by surgical resection. To our knowledge, this was the first report of small bowel DL diagnosed by intraoperative endoscopy in a child.

\section{Case Presentation}

A 15-year-old boy was admitted to the hospital with hematochezia and hypotension. He suffered from epigastric pain, vomiting, and a large amount of hematochezia.
Neither peptic ulcer, nor bleeding disorders were found in the patient or in his family's medical history. Upon arrival in the emergency department, the patient was anemic and ill-looking with complaints of dizziness and dyspnea. His temperature was $36.7^{\circ} \mathrm{C}$, his pulse rate $135 /$ minutes, his respiratory rate $40 /$ minutes, and his blood pressure was $88 / 38$ mm-Hg. On the physical examination, the patient had pale conjunctiva, bounding pulse, and a slightly distended abdomen without tenderness. The liver and spleen were not enlarged. The patient was diagnosed with impending hemorrhagic shock due to massive lower GI bleeding, and was transferred to pediatric intensive care unit.

Hemoglobin, hematocrit, and platelet were $8.1 \mathrm{gm} / \mathrm{dL}$, 25.2\% and 116,000/mm, respectively. Serum transaminases, bilirubin, blood urea nitrogen, creatinine, prothrombin time, partial thromboplastin time, bleeding time, and electrolytes were all normal. The rectal bleeding continued over the next hours. The follow-up values of hemoglobin, hematocrit, and platelet remained low ( $8.3 \mathrm{gm} / \mathrm{dL}, 25.7 \%$, and $104,000 / \mathrm{mm}$, respectively) after transfusion of two units of packed red blood cells, nine units of platelets, and two units of fresh frozen plasma (FFP). Endoscopy and colonoscopy were performed. The endoscopy showed only anemic gastric and duodenal mucosa. The colonoscopy revealed fresh melenic stools covering the mucosa of colon, cecum and terminal ileum; otherwise, an edematous ter- 
minal ileum with scattered polypoid nodular lesions (lymphoid hyperplasia) was observed. The patient received additional four units of packed red blood cells, seven units of platelets, and two units of FFP during the endoscopic examinations. Double-balloon enteroscopy was performed via anus to detect any suspicious lower GI bleeding. However, no active bleeders were found at rectum, colon, and ileum. A subsequent computed tomography (CT) angiography revealed diffuse dilatation of small bowel, colon and rectum, with much blood retention. We observed two contagious well-enhanced nodular lesions at the right upper quadrant of the abdomen on the arterial phase, and we also noticed a leakage of contrast medium into the jejunum on the delayed phase. Therefore, we suspected that the lesions had active bleeding arising from the mucosal surface of jejunum (Figure 1A).

The patient's hemoglobin level dropped to $4.5 \mathrm{mg} / \mathrm{dL}$. He underwent emergency surgical laparotomy. At operation, a small incision of terminal ileum was performed to explore the bleeders by flexible endoscopy. After aggressive endoscopic irrigation of fresh blood and blood clots with normal saline fluid, we found no active bleeder in the ileum. We made another small incision near jejunal-ileal junction, and found a mucosal protruding vascular lesion (approximately $1 \mathrm{~cm}$ in diameter), with pulsatile spurting bleeding at distal jejunum approximately $125 \mathrm{~cm}$ from the Triet'z ligament. After closing the two incisions of ileum, partial bowel resection was performed, and the resected piece revealed that the lesion was a caliber-persistent submucosal dilated artery, approximately $5 \mathrm{~mm}$ in diameter, which was protruding 3-4 $\mathrm{mm}$ from a non-ulcerated mucosal surface (Figure 1B). The patient received four units of packed red blood cells, four units of platelets, and two units of FPP at operation. Histopathology revealed a Dieulafoy lesion of the jejunum. A tortuous, dilated and thickwalled vessel in the submucosa of the small bowel, measuring $5 \mathrm{~mm}$ in diameter was protruded through a small mucosal defect without surrounding inflammatory reaction (Figure 2).

The patient's vital signs became stabilized after operation; his hemoglobin and platelet values rose to $11.5 \mathrm{mg} / \mathrm{dL}$ and $167,000 / \mathrm{mm}$ after transfusion of six units of packed red blood cells and four units of FFP. The patient was advanced to a regular diet two days later and discharged five days after the operation. There was no recurrence of bleeding in the follow-up period.

\section{Discussion}

DL is most often found in the stomach (71\%), followed by duodenum (15\%) and esophagus ( $8 \%$ ). It is uncommon in jejunum, ileum, colon, and rectum (4). DL was observed to be associated with the usage of NSAIDs or anticoagulants, or conditions of alcoholism, stress, or cardiac/pulmonary failure in the elderly $(4,5)$. Mucosal erosion or ischemic injury, which is possibly related to aging and cardiovascular diseases, further weakens the overlying mucosa.

The consensus suggested that DL might rip spontaneously, causing a major bleeding due to the overlying pulsating artery eroded progressively by the mechanical pressure from the abnormal vessel. Histologically, DL is a large three-layered artery with medial hypertrophy, but normal architecture without inflammation, deep ulcerations, and penetration of the muscularis propria, vasculitis, aneurysm formation, or atherosclerosis (1). There was no atherosclerosis or its associated risk factors in our case.

Characteristically, bleeding DLs are presented as acute or recurrent massive GI hemorrhage; there are usually no symptoms of anorexia, dyspepsia, or abdominal pain. The presentation of massive rectal bleeding in our patient was consistent with the reported cases of bleeding DLs in the small bowel (7). Preceding illness of epigastric pain and nausea in our patient might have been due to the large amount of blood retained in the intestines, and an ischemic effect of GI tract by massive blood loss.

Patients with bleeding DL usually present with anemia and postural hypotension, and their mean hemoglobin level on admission has been reported to be between 8.4 -9.2 $\mathrm{g} / \mathrm{dL}$ (8). The average transfusion requirement for the initial resuscitation is usually 3 to 8 units of packed red blood cells (8). Our patient had persistent anemia and hypotension at admission, and needed a major blood transfusion (16 packed red blood cells, 13 units of platelets, and 8 units of FFP within 24 hours) to reach hemodynamic stability. The need for the major blood transfusion in our case was due to a giant DL ( $0.5 \mathrm{~cm}$-diameter of submucosal artery), with acute massive bleeding.

Our case experience confirmed that the diagnosis of patients with DL of the small bowel is often delayed due to inaccessibility and difficulty in localizing the bleeding site. Multidisciplinary approaches such as abdominal CT, radionuclide scan, angiography, capsule endoscopy, and balloon-assisted enteroscopy may help localize the source of bleeding of the small bowel. Angiography is helpful for those patients whose initial endoscopy have failed to detect the bleeding source, while the patient's bleeding is active. The diagnosis of DL of the small intestine can be made angiographically $(2,9)$. The three-phase helical CT angiography could help identify the location of acute lower GI bleeding (10). The diagnosis of the bleeding site was established by CT angiography in $79.2 \%$ (19/24) of the patients (9). The detection of active bleeder by CT angiography was made by observing the contrast enhancement 

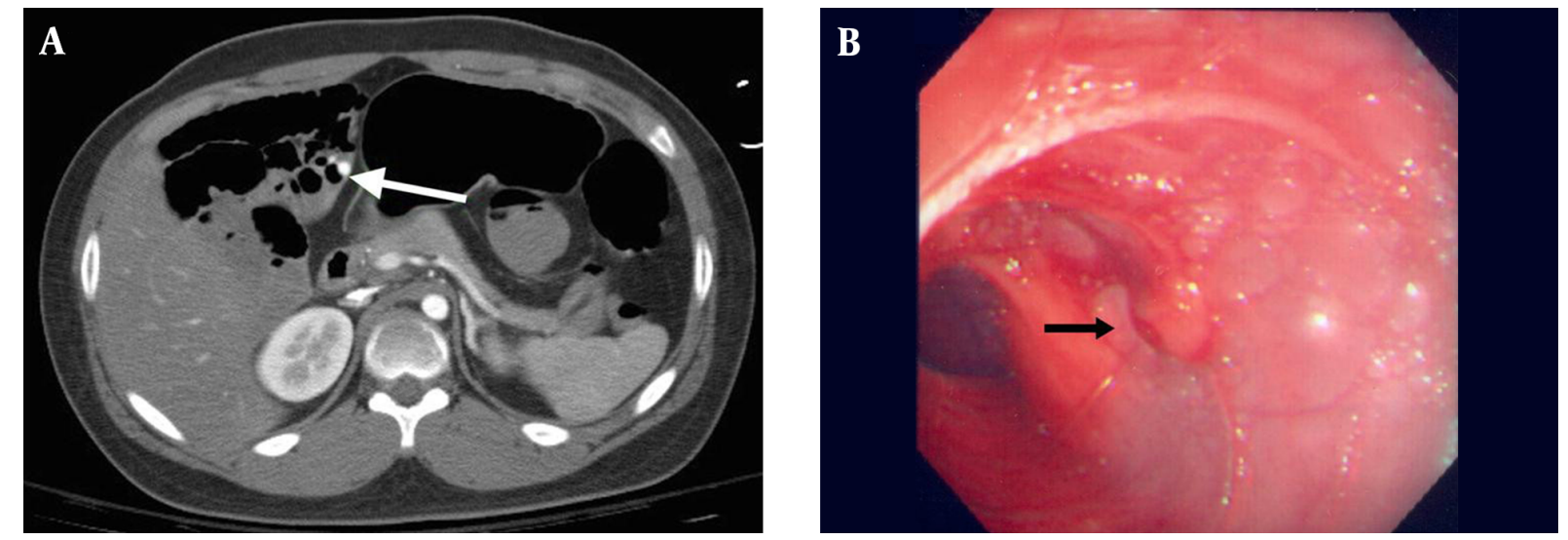

Figure 1. A, CT angiography revealed actively bleeding lesions (arrow) arising from the mucosal surface at jejunum; B, intraoperative enteroscopy by flexible endoscope revealed a protruding, pulsatile vessel (arrow), with active bleeding arising from the mucosal surface at jejunum.

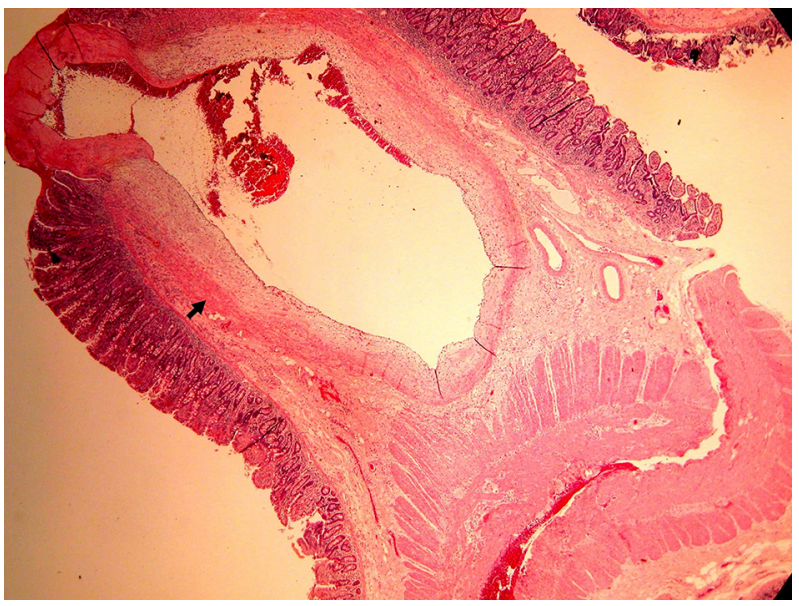

Figure 2. Histology revealed a tortuous, dilated and thick-walled vessel (arrow) in submucosa of the small bowel wall measuring $5 \mathrm{~mm}$ in diameter. It protruded through a small mucosal defect without surrounding inflammatory reaction.

of the bowel wall to evaluate the vascular extravasations of the contrast medium, or thickening of the bowel wall, polyp or tumor, and vascular dilation. In our case, CT angiography clearly identified the active bleeders by observing the enhancement of contrast media during arterial and delayed phases. Small bowel bleeding could be diagnosed and treated directly with the development of balloon-assisted enteroscopy $(11,12)$. In our case, balloonassisted enteroscopy failed to detect the bleeders due to continual active hemorrhage with massive blood clots retained in the small bowel.

If the endoscopic and angiographic approaches fail, surgery must be considered taking into account the patient's clinical condition within an appropriate time. In- traoperative enteroscopy remains a valuable tool in patients with obscure GI bleeding, and it directly evaluates the small intestine, attains a high diagnostic outcome and performs an endoscopic and/or surgical treatment in patients with small bowel hemorrhage $(13,14)$. Consistent with previous experiences (15), DL of the small bowel in our patient could be clearly observed by intraoperative enteroscopy, using flexible endoscope by the incisions of small bowel.

To our knowledge, there were approximately 30 reported cases with jejunal DL based on the literature review, of which only four were about pediatric patients (14, 16, 17). Adult case series reported successful hemostasis with balloon-assisted enteroscopy in patients with DL of the small bowel $(11,12)$. Dulic-Lakovic et al. reported rebleeding in 30\% (3/10) of the patients undergoing balloonassisted enteroscopy, with $20 \%$ (2/10) eventually needing surgical intervention (11). A recent case series $(n=8)$ indicated that balloon-assisted enteroscopy achieved $100 \%$ hemostasis of small bowel DL with low (12.5\%) rate of rebleeding and avoidance of surgery (18). Bleeding DL of the small bowel has been successfully treated by embolization in pediatric patients $(19,20)$. This treatment is usually reserved for patients who do not wish to undergo endoscopic therapy and are poor candidates for surgery.

In a majority of the adult cases, endoscopic hemostasis with balloon-assisted enteroscopy can replace surgery (11-13), while pediatric patients are usually treated with embolization or surgery $(16,17,19)$. In our case, surgery with intraoperative endoscopy was the last and best treatment option due to failure of identifying the bleeder with balloon-assisted enteroscopy and deteriorated hemodynamics. A huge actively bleeding DL indentified by intraoperative endoscopy at jejunum is critical for directing surgi- 
cal therapy.

\subsection{Conclusions}

CT angiography and further surgical intervention with the assistance of intraoperative endoscopy help diagnose the bleeding DL in the small intestine after negative exploration of bleeders in endoscopy, colonoscopy, and doubleballoon enteroscopy. Additional experience with intraoperative endoscopy is a relatively safe and efficient modality for evaluating the bleeding DL of the small intestine.

\subsection{Consent}

Written informed consent was obtained from the patients for publication of this case report and accompanying images.

\subsection{Ethics}

Institutional Review Board of the human research committee at Chang Gung medical foundation approved the study protocol (No.: 201600485B0).

\section{References}

1. Juler GL, Labitzke HG, Lamb R, Allen R. The pathogenesis of Dieulafoy's gastric erosion. Am J Gastroenterol. 1984;79(3):195-200. [PubMed: 6199971].

2. Baxter M, Aly EH. Dieulafoy's lesion: current trends in diagnosis and management. Ann R Coll Surg Engl. 2010;92(7):548-54. doi: 10.1308/003588410X12699663905311. [PubMed: 20883603].

3. Veldhuyzen van Zanten SJ, Bartelsman JF, Schipper ME, Tytgat GN. Recurrent massive haematemesis from Dieulafoy vascular malformations-a review of 101 cases. Gut. 1986;27(2):213-22. [PubMed: 3485070].

4. Schmulewitz N, Baillie J. Dieulafoy lesions: a review of 6 years of experience at a tertiary referral center.Am J Gastroenterol. 2001;96(6):168894. doi: 10.1111/j.1572-0241.2001.03922.x. [PubMed: 11419815].

5. Beyazit Y, Disibeyaz S, Suvak B, Purnak T, Torun S, Parlak E. Evaluation of treatment results among patients with acute gastrointestinal bleeding due to Dieulafoy's lesion admitted to the emergency department [in Turkish]. Ulus Travma Acil Cerrahi Derg. 2013;19(2):133-9. doi: 10.5505/tjtes.2013.58740. [PubMed: 23599197].

6. Sarles HE, Schenkein JP, Hecht RM, Sanowski RA, Miller P. Dielafoy's ulcer: a rare cause of massive gastric hemorrhage in an 11-year-old girl: case report and literature review. Am J Gastroenterol. 1984;79(12):9302. [PubMed: 6334438].

7. Matuchansky C, Babin P, Abadie JC, Payen J, Gasquet C, Barbier J. Jejunal bleeding from a solitary large submucosal artery. Report of two cases. Gastroenterology. 1978;75(1):110-3. [PubMed: 401085].
8. Parra-Blanco A, Takahashi H, Mendez Jerez PV, Kojima T, Aksoz K, Kirihara K, et al. Endoscopic management of Dieulafoy lesions of the stomach: a case study of 26 patients. Endoscopy. 1997;29(9):834-9. doi 10.1055/s-2007-1004317. [PubMed: 9476766].

9. Alshumrani G, Almuaikeel M. Angiographic findings and endovascular embolization in Dieulafoy disease: a case report and literature review. Diagn Interv Radiol. 2006;12(3):151-4. [PubMed: 16972222].

10. Marti M, Artigas JM, Garzon G, Alvarez-Sala R, Soto JA. Acute lower intestinal bleeding: feasibility and diagnostic performance of CT angiography. Radiology. 2012;262(1):109-16. doi: 10.1148/radiol.11110326. [PubMed: 22084211].

11. Dulic-Lakovic E, Dulic M, Hubner D, Fuchssteiner H, Pachofszky T, Stadler B, et al. Bleeding Dieulafoy lesions of the small bowel: a systematic study on the epidemiology and efficacy of enteroscopic treatment. Gastrointest Endosc. 2011;74(3):573-80. doi 10.1016/j.gie.2011.05.027. [PubMed: 21802676].

12. Prachayakul V, Deesomsak M, Aswakul P, Leelakusolvong S. The utility of single-balloon enteroscopy for the diagnosis and management of small bowel disorders according to their clinical manifestations: a retrospective review. BMC Gastroenterol.2013;13:103. doi: 10.1186/1471 230X-13-103. [PubMed: 23800178].

13. Monsanto P, Almeida N, Lerias C, Figueiredo P, Gouveia H, Sofia C. Is there still a role for intraoperative enteroscopy in patients with obscure gastrointestinal bleeding?. Rev Esp Enferm Dig. 2012;104(4):1906. [PubMed: 22537367].

14. Sai Prasad TR, Lim KH, Lim KH, Yap TL. Bleeding jejunal Dieulafoy pseudopolyp: capsule endoscopic detection and laparoscopic-assisted resection. J Laparoendosc Adv Surg Tech A. 2007;17(4):509-12. doi 10.1089/lap.2006.0063. [PubMed:17705738].

15. Kozan R, Gulen M, Yilmaz TU, Leventoglu S, Yilmaz E. Massive lower gastrointestinal bleeding from a jejunal Dieulafoy lesion. Ulus Cerrahi Derg. 2014;30(4):225-7. doi: 10.5152/UCD.2014.2271. [PubMed: 25931935].

16. Moreira-Pinto J, Raposo C, Teixeira da Silva V, Curado J, Barbosa E, Honavar M, et al. Jejunal Dieulafoy's lesion: case report and literature review. Pediatr Surg Int. 2009;25(7):641-2. doi: 10.1007/s00383009-2383-1. [PubMed: 19488764].

17. Marangoni G, Cresswell AB, Faraj W, Shaikh H, Bowles MJ. An uncommon cause of life-threatening gastrointestinal bleeding: 2 synchronous Dieulafoy lesions. I Pediatr Surg. 2009;44(2):441-3. doi: 10.1016/j.jpedsurg.2008.09.033. [PubMed: 19231553].

18. Lipka S, Rabbanifard R, Kumar A, Brady P. A single-center United States experience with bleeding Dieulafoy lesions of the small bowel: diagnosis and treatment with single-balloon enteroscopy. Endosc Int Open. 2015;3(4):339-45. doi: 10.1055/s-0034-1391901. [PubMed: 26356602].

19. Shibutani S, Obara H, Ono S, Kabeshima Y, Kawakubo H, Shito M, et al. Dieulafoy lesion in the ileum of a child: a case report. J Pediatr Surg. 2011;46(5):17-9. doi: 10.1016/j.jpedsurg.2011.01.028. [PubMed: 21616222].

20. Alomari AI, Fox V, Kamin D, Afzal A, Arnold R, Chaudry G. Embolization of a bleeding Dieulafoy lesion of the duodenum in a child. Case report and review of the literature.JPediatr Surg. 2013;48(1):39-41. doi: 10.1016/j.jpedsurg.2012.10.055. [PubMed: 23331838]. 